\title{
Grupo interdisciplinar: construção de Unidade Educacional Sistematizada em Aprendizagem Baseada em Problemas
}

Interdisciplinary group: construction of a Systematized Educational Unit based on Problem-Based Learning

\author{
Ioshie Ibara Tanaka* \\ Sílvia Franco da Rocha Tonhom** \\ Elane de Fatima Taipeiro*** \\ Ana Paula Ceolotto Guimarães **** \\ Maria Yvette de Aguiar Dutra Moravcik***** \\ Teresa Cristina França Sartori ${ }^{* * * * *}$ \\ Elizabete Takeda******
}

\begin{abstract}
Resumo
Em 2003, a equipe de construção da 1a série dos cursos de Medicina e Enfermagem de uma faculdade do interior do estado de São Paulo iniciou um trabalho com objetivo de reconstrução curricular, reestruturando os conteúdos cognitivos e a integração básico-clínica, com o objetivo de se adequar ao Programa de Incentivo às Mudanças Curriculares (PROMED), que propiciou a continuidade das inovações curriculares. Essa equipe trabalhou considerando a dinâmica de grupo, método já utilizado nas sessões de tutoria, e com que os docentes têm certa familiaridade. Para o bom desempenho grupal, os participantes da equipe inseriram-se em dois subgrupos: o de construção de problemas educacionais e o de exercícios de avaliação cognitiva. A equipe e os subgrupos reuniam-se semanalmente, sendo que a cada 15 dias ocorria a socialização dos trabalhos, identificando as fortalezas e/ou fragilidades do processo e dos produtos. A boa adesão, o envolvimento ativo dos participantes e a satisfação manifesta por cada participante com sua inserção no grupo refletiu-se no produto final e contribuiu para a identificação dos sujeitos com a proposta de trabalho. Portanto, nessa trajetória, as transformações individuais e as relações no âmbito da convivência profissional foram determinantes para o processo de reconstrução curricular permanente.
\end{abstract}

Palavras-chave: Aprendizagem Baseada em Problemas. Educação em Saúde. Estudos Interdisciplinares. Equipe de Assistência ao Paciente.

\begin{abstract}
In 2003 the construction team of the 1st grade of the medical and nursing courses from a medical school began a work aiming at curriculum reconstruction, and restructured cognitive contents and the integration of basic and clinical aspects, in order to adapt to the incentive program for Curriculum Changes (PROMED) which allows for the continuity of curricular innovations. This team worked considering the group dynamics, a method already used in tutorial sessions which teachers have some familiarity with. For a good group performance the team participants were divided into two subgroups: one for constructing educational problems and the other for doing cognitive assessment exercises. The team and the subgroups met weekly, and every 15 days the group met for socialization of information, identifying the strengths and weaknesses of the process and/or products. A good adhesion, an active involvement of participants and satisfaction expressed by each participant with their inclusion in the group were reflected in the final product and contributed to the subjects' commitment to the proposal. This way, in the process the individual transformations and relations in a situation requiring the collaboration of professionals were essential in the process of permanent curricular reconstruction.
\end{abstract}

Keywords: Problem-Based Learning. Health Education. Interdisciplinary Studies. Patient Care Team.

\footnotetext{
* Biomédica. Doutora em Análises Clínicas pela UNESP, Campus de Araraquara-SP, Brasil. E-mail: yoibara@hotmail.com

** Enfermeira. Doutora em Educação pela Unicamp-SP, Brasil.

*** Bióloga. Doutora em Ciências: Bioquímica das Adaptações pela UFSCar-SP, Brasil.

**** Farmacêutica / Bioquímica pela USPRP. Doutora em Farmacologia pela FMRP-USP-SP, Brasil.

***** Médica. Especialista em Dermatologia pela HSPESP, Brasil.

****** Bióloga. Doutora em Morfologia pela UNESP, Campus de Botucatu-SP, Brasil.

******* Enfermeira. Doutora em Enfermagem Fundamental pela EEUSPRP-SP, Brasil.
}

As autoras declaram não haver conflitos de interesse. 


\section{INTRODUC̣ÃO}

A faculdade foco da reflexão possui uma história de compromisso com a transformação da educação médica e de enfermagem no Brasil, marcada pela adesão aos projetos Integração Docente Assistencial (IDA) em 1983, Uma Nova Iniciativa na formação de profissionais de saúde (UNI), em 1993 (subsidiado pela Fundação Kellogg), e um Projeto Educacional em 2000.

Esse último promoveu uma significativa reforma curricular com a implementação progressiva de um programa de ensino-aprendizagem centrado no estudante, baseado em problemas $(\mathrm{ABP})$ e orientado à comunidade ${ }^{1}$, direcionando o processo ensino-aprendizagem para o desenvolvimento da capacidade do estudante de construir ativamente seu conhecimento. As atividades curriculares incluíam duas Unidades Educacionais Sistematizadas (UES) baseadas na ABP e Interação Comunitária (IC), que integravam os estudantes de Medicina e Enfermagem. Para implementação dessa nova proposta curricular, tornou-se necessária uma reestruturação administrativa dos cursos, substituindo a tradicional organização por disciplinas em UES, em que cada ano letivo passou a ser gerenciado por um coordenador, e a tarefa de tornar exequível a UES foi destinada aos grupos interdisciplinares, que constituíam as equipes de construção de séries, com funções de elaborar os objetivos específicos e problemas educacionais.

Em 2003, a aprovação pelo projeto institucional $^{2}$ pelo Programa de Incentivo às Mudanças Curriculares - PROMED - propiciou a continuidade das inovações curriculares, no sentido da imagem-objetivo da produção de conhecimento referenciada no modelo de vigilância à saúde, a partir da prática profissional reflexiva, orientada por competência. Esse referencial lançou-nos o desafio de trabalhar um currículo orientado por competência e baseado nas necessidades de saúde da população.

Para alcançar esse desafio, o marco foi a construção da Unidade de Prática Profissional (UPP), que, diferentemente da IC, tem maior inserção e integração com as equipes de saúde e a comunidade, estabelecendo um cenário para a reflexão da prática (aprender fazendo), favorecendo a integração das UES e dos mundos do trabalho e da aprendizagem. A reorientação do modelo de atenção para Necessidades de Saúde, buscando a integralidade do atendimento $^{3}$, viabilizou-se por meio da inserção dos estudantes nas Unidades de Saúde da Família (USF), no Programa da Estratégia de Saúde da Família (PSF), particularmente para os dois primeiros anos dos cursos de Medicina e Enfermagem, inseridos nas equipes multiprofissionais em parceria com a Secretaria Municipal de Saúde de Marília.

Esse enfoque lançou um novo olhar sobre o trabalho da equipe de construção, demandando uma mudança na sua organização e oportunizando a participação de docentes e professores colaboradores inseridos na UPP que contribuíram com o desenvolvimento do referencial teórico de "Necessidades de Saúde". Dessa nova estruturação do trabaIho emergiram diversas questões na equipe de construção, sobre qual seria a inserção dos diferentes conteúdos, bem como o "recorte" de conhecimento delimitado para as diferentes séries. Dentro de tal referencial, a reformulação dos problemas educacionais da UES objetivou uma melhor inserção dos aspectos sócio / antropológicos, bem como da dimensão psicológica e a criação de núcleos familiares abordando o indivíduo, a família e a comunidade.

Essas discussões possibilitaram o avanço da proposta, bem como a reflexão dos profissionais envolvidos sobre sua contribuição e efetiva participação nesse processo. No sentido de fornecer subsídios para a construção do referencial de "Necessidades de Saúde", de contribuir com as reflexões, bem como para qualificação do trabaIho em grupo, contou-se, nesse momento, com uma assessoria externa.

Frente a esse histórico de evolução das mudanças, o presente relato aborda os caminhos da experiência da equipe de construção da UES da $1^{\text {a }}$ série dos Cursos de Medicina e Enfermagem em 2004/2005, com enfoque na dinâmica de grupo e nos fatores efetivos para a constituição dessa equipe. 


\section{RELATO}

Com a implantação do ABP, em 1997, as equipes de construção das UES foram constituídas pelos coordenadores dessas unidades, que indicavam as disciplinas que julgavam necessárias para sua construção. Naquela época, a tarefa de reunir várias disciplinas mostrou-se difícil, uma vez que os docentes ainda estavam se apropriando do método e paralelamente ministrando aulas no método tradicional para as outras séries dos cursos de Medicina e Enfermagem. Oriundos de uma demanda Institucional, aliada à negociação / adesão espontânea à série, algumas vezes os profissionais envolvidos exerciam participações relutantes e pouco efetivas no sentido de articulação e integração de conteúdos. Essa dificuldade refletia-se no produto da equipe, com problemas referenciados no modelo biomédico, pouco integrados e sem articulação entre as dimensões biológica, social e psicológica. Tal situação configurava-se como uma dificuldade constante para a constituição do grupo de trabalho de construção das UES.

A identificação da dificuldade no processo de constituição do grupo propiciou que as mudanças institucionais fossem incorporadas de maneira crítica e com maior clareza dos objetivos do trabalho em grupo.

Para a existência de um grupo, faz-se necessária, mais do que um agrupamento momentâneo de pessoas, uma interação entre elas. Embora existam várias definições de grupo, sua caracterização como "um conjunto de indivíduos, em proximidade física, interagindo, durante certo tempo, para realizar metas comuns, conscientes de que formam uma unidade e que se interinfluenciam" ${ }^{\prime 4}$ explicita os atributos necessários para sua efetiva constituição.

Nos anos de 2004/2005, o grupo de trabaIho constituiu-se de 20 docentes com diversas formações profissionais e atuantes em vários cenários de aprendizagem (tutoria, UPP, conferência, laboratório de atividades práticas e consultoria), tendo um caráter multidisciplinar e multiprofissional.

A constituição do grupo consolidou-se a partir da estruturação do conceito de Necessidades de Saúde, cuja orientação alinhava-se com a Po- lítica Nacional de Humanização (PNH) do SUS, que inclui, entre suas orientações, o parâmetro das ações de saúde centradas nas necessidades de saúde das populações e dos indivíduos ${ }^{5}$.

A diversidade de saberes dos envolvidos na composição grupal favoreceu explorar um mesmo objeto por vários feixes de "luz" ${ }^{6}$, sendo que essa composição espelha a complexidade das situações encontradas na prática que a equipe de construção da UES procurava contemplar nos problemas.

Um dos fatores mais influentes sobre o grupo formal, além de seus indivíduos, é sua administração, no que tange às decisões gerenciais que criam, eliminam ou alteram grupos dentro da estrutura formal da organização ${ }^{7}$.

O processo grupal implica uma rede de relações que pode se caracterizar por relações equilibradas de poder entre os participantes ou pela presença de um líder ou subgrupo que detém o poder e determina as obrigações e normas que regulam a vida grupal. As relações de poder no grupo determinam ou influenciam o grau de participação dos integrantes no processo de comunicação interno, no sistema de normas, nas suas aplicações, punições e decisões ${ }^{8}$.

Kurt Lewin ${ }^{9}$ identificou três tipos de liderança: autocrítica (em que ocorre a total centralização do poder, exercido por coerção), democrática (as decisões são tomadas por maioria; o líder é apenas um representante da vontade de seus liderados) e permissiva (em que é permitido a cada integrante do grupo agir como deseja; não há efetivamente uma ação de liderança). Estudos realizados por diversos psicólogos, levando em conta esses três tipos de classificação, demonstraram que a liderança democrática torna os integrantes do grupo menos dependentes do líder. Já a classificação autocrítica gera maior produtividade, elevando o grau de dependência dos integrantes do grupo em relação ao líder, chegando ao ponto de não saberem produzir sem a sua presença. A liderança permissiva (laissez-faire) gerou os piores resultados.

A liderança é um processo interacional, com características próprias, sendo impossível estabelecer qual a pessoa mais preparada para comandar determinado grupo, uma vez que o líder poderá surgir durante o processo de interação dos participantes. 
Zimerman $^{10}$ destaca como características desejáveis em um coordenador o gostar e acreditar em grupos, ter a capacidade de conter e valorizar as angústias e as necessidades dos outros, bem como suas próprias, ser empático, exercer boa comunicação, possuir senso de humor e a habilidade de integração e síntese.

No ano de 2004, a equipe de construção passou a eleger, entre seus pares, uma dupla coordenação da $1^{\mathrm{a}}$ série (enfermagem e medicina) e seus respectivos suplentes por um ano de mandato.

Ao elegermos nossos coordenadores, reivindicamos sua efetivação nas instâncias organizacionais da Instituiçãa, por entendermos que eles seriam peças-chave para o trabalho do grupo. Tal indicação foi acatada pela Diretoria de Graduação da Faculdade, o que validou a autonomia da equipe de construção e contribuiu para a legitimidade de suas decisões.

Os coordenadores tinham a função de gerenciar o trabalho e a circulação de informações entre os membros do grupo e em outras instâncias organizacionais relacionadas ao trabalho e produção. Com o apoio do grupo, as tarefas de cada coordenador foram determinadas por divisão de trabalho, ficando, esses, responsáveis pela construção dos problemas e conferências, ou pela elaboração dos Exercícios de Avaliação Cognitiva (EAC), análise das questões de aprendizagem, entre outros. Cada um dos coordenadores desempenhava papel-chave no desenvolvimento de cada subgrupo, para torná-lo efetivamente participativo, assegurando a sua totalidade e a individualidade de cada um, nos quais os participantes optaram por um ou outro subgrupo, segundo as prioridades das tarefas, sendo considerada também a diversidade dos profissionais contemplada nos dois espaços.

Após a definição dos participantes do grupo e de seus papéis / atividades foi necessária a estruturação física para a realização das reuniões. Com a organização desse espaço de interação quanto à local, horário e duração, as reuniões ocorriam com periodicidade semanal, em dia e horários fixos, facilitando a participação de cada integrante do grupo.

As reuniões foram fundamentais para o desenvolvimento do próprio grupo, podendo ser definidas como "processo de interação realiza- do por um grupo de pessoas num dado tempo e espaço, no qual se procura atingir os objetivos específicos" (p. 48) ${ }^{11}$.

A equipe de construção contou com uma secretária exclusiva para a série, com as atribuições de manter os arquivos de todos os trabalhos, inclusive de anos anteriores, bem como organizar a logística de contato entre os membros, retorno ao grupo das informações e o controle da lista dos presentes nas reuniões.

O desenvolvimento dos trabalhos dava-se de forma independente, no primeiro momento, sendo que, depois do cumprimento de tarefas definidas previamente, voltava-se ao grupo todo para a socialização e possíveis alterações, se necessário fosse. Além de otimizarmos o cumprimento das tarefas, a divisão manteve um número adequado de participantes em cada subgrupo, pois existe um tamanho limite para um grupo, que é aquele que não interfira negativamente na comunicação ${ }^{10}$.

Importante destacar que, nas reuniões para o compartilhamento das atividades desenvolvidas nos subgrupos, os membros elegiam um coordenador para essas reuniões, com a função de desenvolver a agenda do dia, ordenar as discussões, bem como gerenciar o tempo. Considerou-se ser importante a troca de papéis no sentido de valorização de cada um, bem como a possibilidade de experiências diversas dentro do processo grupal.

Um fator relevante que emergiu das relações pessoais no grupo foi a incorporação da humanização neste processo grupal, à semelhança do que esperávamos construir em nossa prática quanto às atividades dos estudantes na série no que tange à $\mathrm{PNH}^{12}$.

Segundo Pichon-Rivière ${ }^{13}$, existem vetores universais de avaliação para compreender-se ou analisarem-se os processos grupais: afiliação (grau de identificação dos integrantes entre si e com a tarefa), pertença (maior grau de identificação e integração com o grupo, permitindo a elaboração de tarefas e estratégias), cooperação (desempenho de papéis diferenciados e complementares, favorecendo a participação e contribuição para a tarefa grupal), pertinência (foco no esclarecimento da tarefa prescrita e no seu cumprimento), comunicação (intercâmbio de significados, podendo ser verbal ou não-verbal) e a 
aprendizagem (ocorre a resolução de ansiedades, criação, adaptação ativa à realidade, envolvendo reestruturação e redistribuição de papéis entre os integrantes do grupo). Ainda segundo o mesmo autor, a comunicação é o mais importante, podendo ser a indicativa de como estão operando os demais vetores.

Um diferencial importante para o grupo foi os participantes identificavam os aspectos positivos e negativos do trabalho desenvolvido e colocavam sugestões para sua resolução de forma espontânea. Identificou-se um bom nível de participação dos docentes nas reuniões, atribuindo-se isso ao fato dos indivíduos sentirem-se parte do grupo. Esse momento avaliativo proporcionou a valorização de cada participação e reflexão sobre a responsabilidade individual quanto aos objetivos e propostas do trabalho, bem como nas decisões tomadas pelo grupo. Diversos autores enfatizam a importância e o caráter dinâmico da avaliação no trabalho grupal, considerada fundamental para uma constante adequação do trabalho ${ }^{14}$.

Acredita-se que o processo avaliativo foi o canal efetivo de comunicação no grupo, que, associado à confiança e ao compromisso, minimizaram-se os conflitos e aumentou-se a produtividade grupal. A avaliação forneceu o fio condutor nos momentos em que o grupo sentia-se improdutivo, divergente ou mesmo com leituras implícitas e distorcidas sobre algum membro ou trabalho realizado. Ainda, segundo Giffoni, "comunicar é colocar ideias em comunhão, e tornar comum não significa concordar, pois também há comunicação na discordância e na diferença" (p. 3) $)^{15}$. Portanto, esse fio permitiu a cooperação na superação dos obstáculos e explicitou as diferenças como algo fundamental para a identidade do grupo.

A humanização, enquanto política, possui ainda como um de seus princípios a transversalidade, que se configura como um modo de estruturar as relações baseadas no reconhecimento da alteridade e do diálogo. Isso pressupõe a busca por relações profissionais que valorizem o investimento na formação humana, esforço pela compreensão por diferentes pontos de vista e tolerância, bem como discussão de conflitos e

contradições. A humanização é indispensável em todas as relações e, no grupo, configura-se como o respeito pela contribuição do outro e pela abertura de canais de diálogo. O grupo buscou incorporar tais premissas, abrindo a avaliação de maneira formativa e construtiva, quanto às suas participações, contribuições e sugestões, com respeito pelo outro e seus valores, bem como suas fragilidades. Emergem dessa postura desafios como o reconhecimento da pessoa, ou seja, o sentido de ser membro, e o de sujeito, ou seja, de sua identidade, com o necessário respeito a outros referenciais pessoais. Embora o grupo se configure como executor de uma tarefa, as identidades e singularidades respeitadas aumentam o sentimento de pertença e consequente colaboração e investimento pessoal para realizar as tarefas, transformando a práxis ${ }^{16}$.

Zimerman ${ }^{10}$ considera que em todos os grupos coexistem duas forças contraditórias, a coesão e a desintegração. À medida que cada membro se sinta parte efetiva do grupo e defenda os objetivos do mesmo, a coesão prevalece.

Por outro lado, dada a natureza e missão dessa equipe de construção, um requisito básico para sua existência é que ela atenda aos objetivos institucionais, sendo maior a eficácia deste grupo quanto maior for sua integração ${ }^{17}$.

Portanto, para que, efetivamente nos constituíssemos em um grupo, ou seja, prevalecesse a coesão, foi necessário que ocorresse a identificação com as propostas e diretrizes que nortearam o rearranjo de papéis e atribuições oriundas das mudanças Institucionais. Essa sintonia entre missão Institucional e expectativa individual foi alcançada quando a comunicação, efetivamente estabelecida nesse espaço de manifestação grupal, estruturou a interação entre as diferentes visões e expectativas, fortalecendo as relações e identificação com a proposta de trabalho para a $1^{\text {a }}$ série.

\section{CONCLUSÃO}

Considerando-se as interações necessárias para que se tenha um novo olhar sobre o trabalho de um grupo, acreditamos que a estruturação alcançada favoreceu o estabelecimento de tarefas efetivas e a cooperação entre seus membros, o 
que deriva da percepção individual de pertencer a um grupo.

Embora se reconheça o caráter único de cada grupo, dada sua evolução particular, derivada da individualidade de seus membros, de seus valores e motivações, acreditamos que a experiência relatada permitiu evidenciar as características fundamentais como o respeito às diferenças, tanto em relação às áreas de conhecimento de cada profissional quanto às aproximações com o novo referencial adotado; como as atitudes de querer desvendar o "novo" mesmo que a cada revelação as angústias se acentuavam; como a humanização como modo de estruturar as relações baseadas no reconhecimento da alteridade e do diálogo; como a identificação e a aceitação no grupo, que foram essenciais na valorização de cada membro da equipe. Todos esses aspectos tornaram a vivência grupal um momento produtivo e prazeroso, contribuindo para sua efetiva constituição.

A opção pelo trabalho em grupo e/ou em subgrupo também refletiu a necessidade de interação e troca dinâmica de informações, experiências e rápido "feedback" entre os responsáveis pelos conteúdos pertinentes àquela UES, estruturando-se como um espaço coletivo de criação, refletindo positivamente na formação de novos grupos de trabalho que foram se formando para a construção de novas mudanças que vêm acontecendo até o presente momento.

\section{REFERÊNCIAS}

1. Manual Famema. Faculdade de Medicina de Marília; 1997.

2. Promed. Uma nova escola médica para um novo sistema de saúde: projeto final da FAMEMA. Marília; 2003. Disponível em: http://www.famema.br/institucional/documentos/promed final.pdf

3. Cecílio LCO. As necessidades de Saúde como conceito Estruturante na Luta pela Integralidade e equidade na Atenção em Saúde. In: Pinheiro R, Mattos, RA, organizadores. Os sentidos da integralidade na atenção e no cuidado à saúde. Rio de Janeiro: IMS/UERJ, ABRASCO; 2001. p. 113-27.

4. Molina MIG. Fundamentos para o trabalho com grupos em extensão rural. Cad Dif Tecnol. 1988;5(1/3):77-95.

5. Deslandes SF. O projeto ético-político da humanização: conceitos, métodos e identidade. Interface Comunic Saúde Educ. 2005;9(17):389-406.

6. Rigobello LMM, Fortuna CM, Rigobello LX, Pereira MJB, Diniz SA. Processo de Comunicação em Grupos de Aprendizagem: uma experiência multiprofissional. Rev Latino-Am Enferm. 1998;6(4):95-102.

7. Amaru ACO. Gerência do Trabalho em Equipe. Pioneira; 1986.

8. Alexandre M. Breve descrição sobre processos grupais. Comum. 2002;7(19):209-19.

9. Lewin K. Resolvendo conflitos sociais: trabalhos relacionados na dinâmica de grupo. Gertrude W Lewin. New York: Harper \& Row; 1948.

10. Zimerman DE. Fundamentos básicos das grupoterapias. Artes Médicas Sul. 1997;5:51-4.

11. Jay A. How to run a meeting. Harvard Business Review. 1976;54(2):43-57.

12. Brasil. Ministério da Educação. Conselho Nacional de Educação. Parecer CNE/CES 1.133/2001, de 7 de agosto de 2001: Diretrizes curriculares nacionais dos cursos de graduação em enfermagem, medicina e nutrição. Brasília; 2001. Disponível em: http://portal.mec.gov.br/dmdocuments/ces1133.pdf

13. Pichon-Rivière E. O processo grupal. Trad Marco Aurélio Fernandes Velloso. Martins Fontes: São Paulo; 1994.

14. Contreras JM. Como trabalhar em grupo: introdução à dinâmica de grupos. São Paulo: Paulus Editora; 1999.

15. Giffoni VL. Comunicação. São Paulo; 1989. 5 p. (mimeo)

16. Rego S, Gomes, AP, Siqueira-Batista R. Humano demasiado humano: bioética e humanização como temas transversais na educação médica. Cadernos ABEM. 2007;3:24-33.

17. Rodrigues MVC. Grupos de Trabalho: dos conceitos clássicos à panacéia. Rev Tendência Trabalho. 1996;29-34. 